\title{
Reduced Effectiveness of Standard Recruitment for Deceased Organ Donor Registration: The Need for Population-Specific Recruitment Materials
}

\author{
Patricia Zheng • Rachel Kornfield • Cathy Olmo • \\ Jennifer Guy $\cdot$ John Inadomi $\cdot$ Scott W. Biggins
}

Received: 28 July 2010/ Accepted: 23 December 2010/Published online: 9 January 2011

(C) The Author(s) 2011. This article is published with open access at Springerlink.com

\begin{abstract}
Background The low rate of deceased donor organ donation limits the availability of life-saving transplants. Transplant candidate caregivers are an under-utilized but potentially devoted pool of advocates who themselves may be recruited to register for deceased organ donation.

Aims To compare the effectiveness of recruitment materials in Transplant Candidate Caregivers (TCC) and San Francisco Bay Area Health Fair Attendees (HFA).

Methods Each subject was given a California Transplant Donor Network educational pamphlet and cohort-coded registration materials. The primary outcome was the
\end{abstract}

\footnotetext{
P. Zheng $\cdot$ R. Kornfield $\cdot$ J. Guy

Division of Gastroenterology, University of California

San Francisco, San Francisco, CA, USA

e-mail: patricia.zheng@ucsf.edu

R. Kornfield

e-mail: rkornfield@uchicago.edu

J. Guy

e-mail: jennifer.guy@ucsf.edu

C. Olmo

California Transplant Donor Network, Oakland, CA, USA

e-mail: colmo@ctdn.org

J. Inadomi

Division of Gastroenterology, University of Washington,

Seattle, WA, USA

e-mail: JInadomi@medicine.washington.edu

S. W. Biggins $(\bowtie)$

Division of Gastroenterology, University of Colorado Denver, 1635 Aurora Court MS B154, 7th Floor AO Pavilion, Aurora, CO 80045, USA

e-mail: scott.biggins@ucdenver.edu
}

number of new registrations per recruitment packet distributed.

Results A total of 232 recruitment packets were distributed; 116 to each of the two cohorts. The TCC group was more likely to be older ( 49 vs. $45, p=0.05$ ), female ( 71 vs. $63 \%, p=0.2$ ), Hispanic ( 21 vs. $5 \%, 0.001)$, married (75 vs. $33 \%, p<0.0001)$, and less educated $(p=0.007)$. Despite demographic differences, the two groups had similar prior donor registration rates $(40 \%$ TCC vs. $50 \%$ HFA, $p=0.11$ ). However, with a minimum 2-week follow-up, the number of new registrations was only nine in the TCC cohort as compared to 38 in the HFA cohort (0.33 vs. 0.80 new registrations/packet, $p<0.0001$ ).

Conclusions The effectiveness of standard deceased donor registration recruitment materials is reduced in Transplant Candidate Caregivers as compared to Health Fair Attendees. This reduced efficacy may be due to dissimilar demographics, psychosocial status at time of recruitment, and beliefs about organ donation. Development of audience specific recruitment materials may improve efforts to register Transplant Candidate Caregivers for deceased organ donation.

Keywords Tissue and organ procurement - Organ donor · Recruitment · Organ transplantation · Registration

\section{Introduction}

The number of life-saving organ transplants performed in the United States is limited by the shortage of available donor organs. As need outstrips supply, the number of patients waiting and dying on the waiting list is increasing $[1,2]$. The majority of available organs come from donations after death, and since the decedent's preference is one 
of the strongest predictors of actual donation [3, 4], it is important for individuals to make their donation intent known by joining organ donation state registries through first-person consent $[5,6]$.

Encouragingly, since state registries were established in the early 2000s, donor designation rates have continually increased, and 86.3 million people have enrolled in donor registries nation-wide [7]. However, despite such progress, only $25 \%$ of registry eligible Californians are currently registered [8]. Donor designation rates vary significantly between racial groups and according to county of residence and ethnicity, suggesting that socioeconomic status and cultural environment may influence perceptions and acceptance of organ donation and transplant.

Identification of specific groups receptive to becoming organ donors and understanding the factors that influence registration decisions can increase the success of recruitment efforts. Additional analysis of why members of certain groups choose not to donate can facilitate the design of tailored strategies for overcoming their reluctance [9]. An example of successful implementation of this strategy occurred in high schools students. Given high school students' historic low levels of knowledge about organ donation, and early studies that identified knowledge can predict a favorable attitude towards donation [10], targeted organ donation education programs were initiated in selected high schools and subsequently shown to improve both knowledge of and attitudes toward donation in students $[11,12]$. The success of this strategy suggests that identifying receptive groups and devising interventions to specifically address barriers to donation may prove to be important in increasing new donation registrations.

One group of individuals who may be particularly receptive to recruitment is the caregivers of potential transplant candidates. Researchers have previously hypothesized that caregivers' personal experiences with patients in need of organ transplantation and greater awareness of the process may make them more receptive to recruitment $[13,14]$. Additionally, this group of individuals has already been crucial to the outreach recruitment efforts of local organ procurement organizations, often volunteering as speakers on behalf of the topic and representatives at recruitment events. Given the frequency of their clinical visits, they are also readily targetable through flyers left at transplant center offices and waiting rooms. We hypothesized that transplant candidate caregivers would be particularly motivated to register and to recruit others to register as deceased organ donors when compared to the general public. The aim of this study was to evaluate the effectiveness of standard recruitment materials in two groups: (1) transplant candidate care givers and (2) health fair attendees, the target population of current recruitment efforts by organ procurement organizations.

\section{Methods}

Study Population and Patient Recruitment

In this study, we obtained English-language organ donor registration materials and educational pamphlets from our organ procurement organization, the California Transplant Donor Network (CTDN) (Fig. 1). The standard CTDN educational pamphlet contains a brief description of the organ donation process, a list of donation facts, and an explanation of the various methods of organ donor registration. Registration materials contained instructions for on-site paper registration, mail-in registration, and Webbased registration. All registration materials were coded by cohort. Prior to intervention, donor registration status of the subjects, as well as demographic data, was tracked on an individual level. New registrations made by subjects and associates with whom the subjects shared their materials were counted by CTDN and tracked only on a cohort level.

Educational and registration materials were given to two distinct cohorts: (1) transplant candidate caregivers (TCC) accompanying liver and/or kidney transplant candidates to transplant educational classes at the University of California San Francisco Transplant Clinic and (2) health fair attendees (HFA) in the San Francisco Bay area. Materials were provided in a standardized manner by the study investigators (PZ, RK). All subjects were required to be English-speaking, at least 16 years of age, and residents of California. Each subject receiving organ donation registration materials was asked to complete a 13-item demographic questionnaire that also assessed whether or not the subject possessed a driver's license or had previously registered as a deceased organ donor. Subjects were encouraged to consider registering as organ donors and to share their education and registration materials with members of their family and social network. No compensation was given to subjects for participation in the study. When multiple caregivers accompanied a transplant candidate to the educational class, materials were given only to one caregiver. Materials were distributed regardless of prior registration status. Recruitment of and registrations resulting from both cohorts occurred from August 2009 through January 2010. The study was approved by the UCSF Committee on Human Research.

\section{Statistical Methods}

Subject characteristics were tabulated and compared using a $t$ test for continuous variables and Chi-square or Fisher's exact test for categorical variables. Effectiveness of recruitment materials was assessed by comparing the proportions of new registrants per recruitment packet 

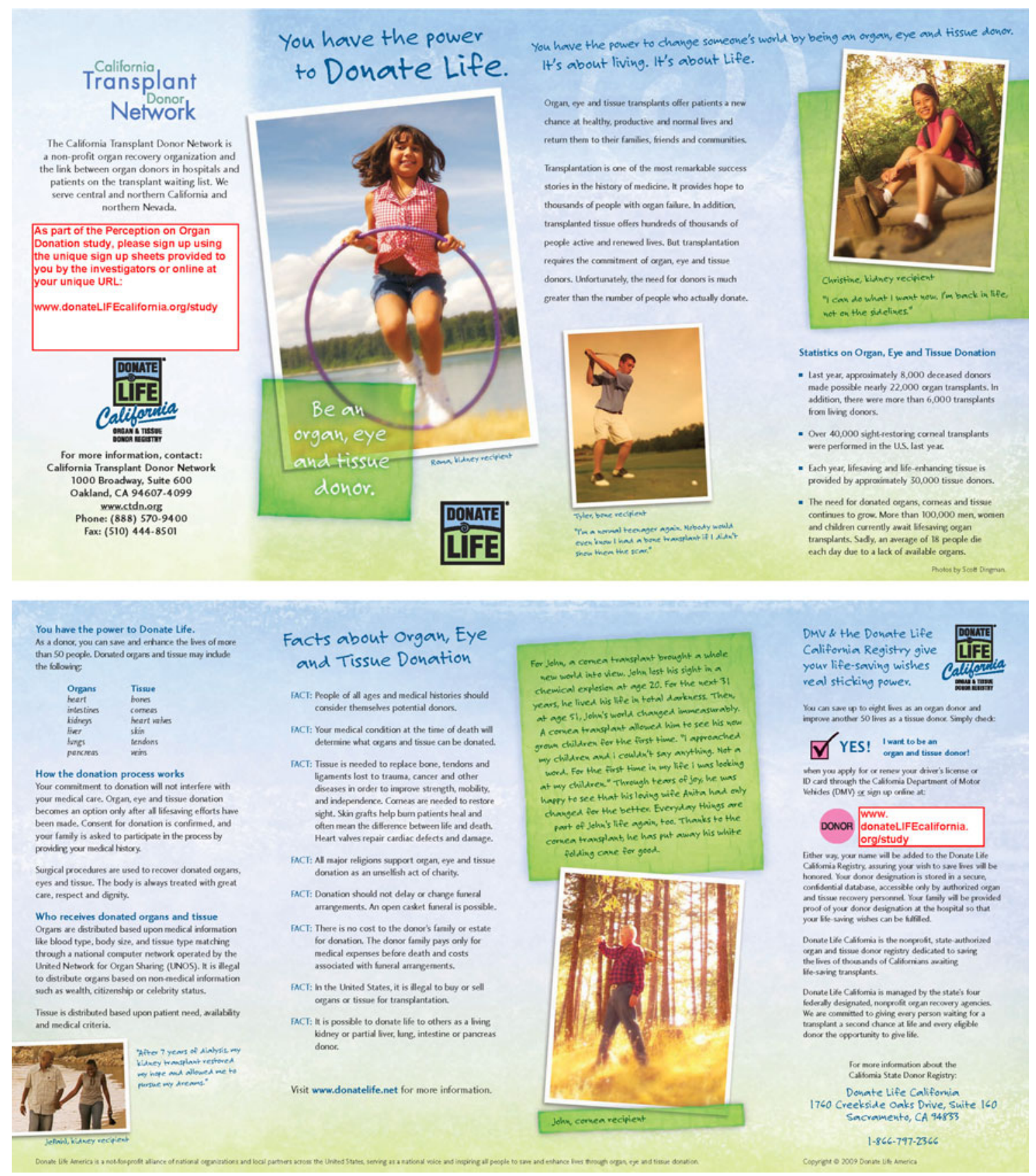

Fig. 1 Standard CTDN donation informational pamphlet

distributed in the two cohorts. All statistical analysis was preformed using STATA version 11.0 (STATA Corp, College Station, TX).

\section{Results}

A total of 232 donor recruitment packets were distributed; 116 to the TCC cohort and 116 to HFA cohort. The demographics of the subjects are shown in Table 1. The
TCC cohort was similar to the HFA cohort in terms of percent with active religious participation (42, 36\%, $p=0.35)$ and percent with a driver license (93, 88\%, $p=0.18)$. However, the TCC cohort was more likely to be older (49 years old vs. $45, p=0.024$ ), female (71 vs. $63 \%$, $p=0.2$ ), Hispanic (21 vs. $5 \%, p=0.001$ ), married (75 vs. $33 \%, p<0.0001)$, and less educated $(p=0.007)$. The cohorts also differed in race. The HFA cohort was predominantly Caucasian, whereas the TCC cohort had a higher proportion of Asians (Caucasian 43 vs. 63\%, $p<0.001$; Asian 38 vs. 20\%, $p=0.036$ ). 
Table 1 Subject characteristics

\begin{tabular}{|c|c|c|c|c|c|}
\hline & \multicolumn{2}{|c|}{ Transplant Candidate Caregivers (TCC) } & \multicolumn{2}{|c|}{ Health Fair Attendees (HFA) } & \multirow[t]{2}{*}{$p$ value } \\
\hline & $\#$ & $\%$ of total & $\#$ & $\%$ of total & \\
\hline Sample size $(n)$ & 116 & - & 116 & - & \\
\hline Average age & 49 & - & 45 & - & 0.024 \\
\hline Female & 82 & $71 \%$ & 73 & $63 \%$ & 0.210 \\
\hline Hispanic & 24 & $21 \%$ & 6 & $5 \%$ & 0.001 \\
\hline \multicolumn{6}{|l|}{ Race } \\
\hline Caucasian & 42 & $43 \%$ & 73 & $63 \%$ & $<0.001$ \\
\hline African American & 12 & $12 \%$ & 15 & $13 \%$ & 0.54 \\
\hline American Indian & 4 & $4 \%$ & 5 & $4 \%$ & 0.74 \\
\hline Asian or Pacific Islander & 37 & $38 \%$ & 23 & $20 \%$ & 0.036 \\
\hline Other race & 3 & 3 & 0 & 0 & 0.081 \\
\hline Highest education level & & & & & $0.007 *$ \\
\hline High school & 37 & $32 \%$ & 22 & $19 \%$ & - \\
\hline Some college & 75 & $67 \%$ & 94 & $81 \%$ & - \\
\hline Married & 87 & $75 \%$ & 38 & $33 \%$ & $<0.001$ \\
\hline Has children & 30 & $63 \%$ & 32 & $43 \%$ & 0.90 \\
\hline Regularly attends religious services & 49 & $42 \%$ & 42 & $36 \%$ & 0.35 \\
\hline Driver license & 108 & $93 \%$ & 102 & $88 \%$ & 0.18 \\
\hline Previously registered for deceased organ donation & 46 & $40 \%$ & 58 & $50 \%$ & 0.11 \\
\hline
\end{tabular}

$n$ number, $S D$ standard deviation; *Differenced tested by ANOVA

There was no statistically significant difference in the numbers of TCC and HFA already registered as organ donors (40 vs. $50 \%, p=0.11$ ). Across the two cohorts, age, sex, or race were not associated with prior registration, but there was a trend toward lower prior registration rates among Hispanics $(p=0.078)$. When compared to Caucasians, Asians were less likely to already be registered as organ donors in the TCC cohort $(p=0.016)$.

With at least a 2-week follow-up period, there were a total of nine new registrations in the TCC cohort and 38 in the HFA cohort (Table 2). The majority of the new registrants in both the TCC and HFA cohorts registered on-site using a paper sign up form (seven among the TCCs, and 29 among the HFAs). A total of seven TCCs and 34 HFAs registered through paper forms, and two of the TCCs and four of the HFAs signed up online via the unique URL provided on the recruitment pamphlet. The rate of overall new registrations was lower in the TCC cohort compared to the HFA cohort ( 0.8 vs. 0.33 new registrations/packet, $p<0.0001)$.

\section{Discussion}

The availability of life-saving organ transplant procedures is limited by the shortage of donor organs. The scarcity of organs for transplantation has compelled the transplant community to look for ways to safely expand the organ donor pool, which has included efforts to encourage enrollment in state organ donor registries. Improving rates of first-person consent for deceased organ donation can save lives by increasing the availability of donor organs for patients waiting for transplants.

Table 2 Effectiveness of recruitment materials

\begin{tabular}{|c|c|c|c|c|c|}
\hline \multirow[b]{2}{*}{ New registrations type } & \multicolumn{2}{|c|}{ Transplant Candidate Caregivers (TCC) } & \multicolumn{2}{|c|}{ Health Fair Attendees (HFA) } & \multirow[t]{2}{*}{$p$ value } \\
\hline & \# & $\%$ cohort & $\#$ & $\%$ cohort & \\
\hline On-site paper & 7 & $6 \%$ & 29 & $25 \%$ & $<0.001$ \\
\hline Mail in paper sign ups & 0 & - & 5 & $4 \%$ & 0.024 \\
\hline Online sign ups & 2 & $2 \%$ & 4 & $3 \%$ & 0.408 \\
\hline Total new registrations & 9 & $8 \%$ & 38 & $33 \%$ & $<0.0001$ \\
\hline New registrations per recruitment packet & 0.33 & - & 0.80 & - & $<0.0001$ \\
\hline
\end{tabular}


This is the first study to track the efficacy of standard organ donation recruitment materials. We assessed the overall effectiveness of the standard education material packet distribution to generate new registrations both directly and indirectly by motivating two groups of potential community advocates - the Transplant Candidate Caregivers and the Health Fair Attendees. We distributed one recruitment packet to each individual in the Transplant Candidate Caregiver and the Health Fair Attendees cohorts irrespective of their prior registration status. In total, distribution of 232 recruitment packets to 104 previously unregistered and 128 previously registered individuals yielded 47 new registrations. On an intent-toregister basis, defined as the number of new donor registrations yielded from the recruitment packets distributed to each cohort, we found a significantly reduced effectiveness in the transplant care givers compared to the health fair attendees, 0.33 versus 0.80 new registrations per packet, respectively ( $p<0.0001)$. Since no prior study has analyzed the efficacy of donor registration recruitment in this manner, the relative success of these recruitment efforts to other efforts outside this study cannot be assessed. However, considering the brevity of the interaction involved in handing out pamphlets at health fairs and in clinics, the outcomes seem to justify the recruiter's time and efforts. For some subjects, reminding them of the option of deceased donor organ registration and providing sign up materials was a sufficient strategy. For those who did not sign up, it is unclear whether their reluctance stems from negative attitudes about donation, lack of knowledge, or other factors.

Marketing research suggests that when marketing products like the concepts of organ donation registration, which offer little direct benefit to the consumer, it is important to identify specific populations receptive to targeted recruitment and to address their specific barriers to donation [15]. Our study evaluated two groups, the HFAs, the current targets of most donation recruitment by organ procurement organizations, and the TCCs, a previously uncharacterized group that we hypothesized would be particularly receptive to organ donation registration recruitment efforts given their personal knowledge of the scarcity of donor organs through their interactions with transplant candidates and medical teams. We hypothesized that caregivers of transplant candidates would be more willing to register themselves and to motivate their friends and family to register as deceased donors. However, we found that rates of new first-person consent for organ donation were lower in the TCC cohort than in the general population attending health fairs, despite similar rates of registration prior to our intervention.

There are several possible explanations for the reduced efficacy of recruitment materials in the TCC cohort.
Previous research has looked at the effects of an individual's race [16], ethnicity [17], and attitude about donation [18] on willingness to donate. These studies found that some of the demographic characteristics that were more pronounced in the TCC cohort, i.e., being more Hispanic, Asian, female, and less educated, have been associated with less favorable attitudes toward organ donation and willingness to register as organ donors [3, 19-22]. This reduced willingness to register may be due to increased suspicion of the medical community in some minority populations [23]. Additionally, since social networks often consist of individuals of similar backgrounds [24], the differential willingness to donate between the cohorts might have been compounded when study subjects attempted to recruit friends or family.

Alternatively, the reduced efficacy of standard non-targeted recruitment efforts in the TCC cohort may be attributable to other factors associated with the transplant evaluation process and setting that limit the receptiveness of TCCs to recruitment. Our study intervention took place early in the transplant education process, at a time when the transplant candidates and caregivers are suffering emotional stress from adjusting to the new diagnoses of chronic end stage organ disease. Studies have previously reported that TCCs suffer from high levels of stress because of their caregiver roles [25]. This psychological burden might inherently preclude them from thinking about donor registration both because of lack of time and the weight of the level of responsibility for and proximity to a seriously ill loved one. Yet, since many of the organ procurement organizations' most ardent supporters and advocates are the caregivers or previous caregivers of transplant candidates, we believe it is possible that subsequent outreach effort to the TCCs later in the transplant candidates' medical care (e.g., after a successful transplant) might yield different recruitment effectiveness.

Whether the factors that influence the decision to donate differ between the transplant candidate caregivers and the general public has not been investigated; however, further identification of the specific factors that influence the TCCs and HFAs in their organ donation registration decisions will help elucidate the observed differences in recruitment effectiveness and provide ways to optimize recruitment strategies. Indeed, previous research has shown that tailored, population-specific approaches to recruitment that addressed individual beliefs and barriers are more efficient in directing behavior than more broadly aimed generic population recruitment strategies [26, 27]. When recruitment efforts are targeted to the specific characteristics and needs of transplant candidate caregivers, we may increase their receptiveness to registering and harness their true capacity as advocates for deceased donor organ donation. 
While prior studies have assessed groups' stated willingness to donate their organs after death, to our knowledge, this is the first study to measure the actual rates of new registrations. Since it has not been possible to assess the success of organ registry recruitments because of the ethical and empirical issues raised by tracking individual registrations, the use of cohort-coded paper and online sign-up forms in this study provides a novel way of studying the effectiveness of organ donation registration recruitment. Unlike traditional surveys focusing on willingness to be register as an organ donor, our study measured actual registration activity. We expect that application of this method in future studies to optimize tailored targeting approaches in targeted populations reluctant to register to become organ donors may optimize outreach efforts.

Our study has limitations. We were unable to track individual level data on efficacy of recruitment materials because of the need to preserve subject anonymity. We were therefore unable to perform an analysis of the individual psychosocial factors that might influence an individual's likelihood to register and ability to recruit others to register. Additionally, we were unable to differentiate the number of registrations between direct participants and the social networks of participants. Further separation of the registration materials into additional tracking categories in the future can help differentiate between sign-ups from direct participants and their social networks. Our current study used an outreach model with in-person packet distribution; it may be of interest to also assess the effectiveness of more passive packet distribution without an onsite in-person advocate such as a clinic waiting room or department of motor vehicle office waiting area. Finally, our study mainly tested the effectiveness of recruitment practices, and we did not gather data on the attitudes and beliefs that motivated each cohort's decision-making about organ donation. Such investigations might help explain the observed differences in recruitment success by exploring the reasons that individuals in these groups choose whether or not to register as organ donors.

Overall, this study assessed not only the willingness to donate but also the effectiveness of recruitment materials in a previously uncharacterized group of caregivers of potential transplant patients (the TCC group). Surprisingly, we found that TCCs were less likely to register as firstperson consent organ donors and were less receptive to generic deceased donor registration recruitment materials. It is possible that through tailoring our recruitment strategy, including modifying how and where these individuals are approached, that this group could be successfully motivated to register themselves and to become advocates encouraging the registration of others. Further research into the attitudes and beliefs that contribute to decisions to provide first-person consent for deceased organ donation could facilitate development of targeted recruitment strategies with the goal of increasing donation rates.

Acknowledgments This work was funded in part by grants from the National Center for Research Resources (KL2 RR024130), from the National Institute of Diabetes and Digestive and Kidney Diseases (DK076565) and from Agency for Healthcare Research and Quality (DK076565).

Open Access This article is distributed under the terms of the Creative Commons Attribution Noncommercial License which permits any noncommercial use, distribution, and reproduction in any medium, provided the original author(s) and source are credited.

\section{References}

1. Port FK, Dykstra DM, Merion RM, Wolfe RA. Organ donation, transplantation trends in the USA, 2003. Am J Transplant. 2004;4:7-12.

2. Wolfe RA, Merion RM, Roys EC, Port FK. Trends in organ donation and transplantation in the United States, 1998-2007. Am J Transplant. 2009;9:869-878.

3. Siminoff LA, Gordon N, Hewlett J, Arnold RM. Factors influencing families' consent for donation of solid organs for transplantation. JAMA. 2001;286:71-77.

4. Rodrigue JR, Cornell DL, Howard RJ. Organ donation decision: Comparison of donor and nondonor families. Am J Transplant. 2006;6:190-198.

5. Koop CE. Increasing the supply of solid organs for transplantation. Public Health Rep. 1983;98:566-572.

6. Morgan SE, Miller JK. Communicating about gifts of life: The effects of knowledge, attitudes, and altruism on behavior and behavioral intentions regarding organ donation. J Appl Commun Res. 2002;30:163-178.

7. National Donor Designation Report Card. Cathy Olmo, Community Development Supervisor, California Transplant Donor Network. Personal communication. 2010; 18 June 2009.

8. California Transplant Donor Network. Unpublished Internal Report. Cathy Olmo, Community Development Supervisor, California Transplant Donor Network. Personal communication, 18 June 2009.

9. Prottas JM. Encouraging altruism: Public attitudes and the marketing of organ donation. Milbank Mem Fund Q Health Soc. 1983;61:278-306.

10. Horton RL, Horton PJ. Knowledge regarding organ donation: Identifying and overcoming barriers to organ donation. Soc Sci Med. 1990;31:791-800.

11. Weaver M, Spigner C, Pineda M, Rabun K, Allen M. Knowledge and opinions about organ donation among urban high school students: Pilot test of a health education program. Clin Transplant. 2000;14:292-303.

12. Reubsaet A, Brug J, Nijkamp MD, et al. The impact of an organ donation registration information program for high school students in the Netherlands. Soc Sci Med. 2005;60:1479-1486.

13. Jacob Arriola KR, Robinson DHZ, Perryman JP, Thompson N. Understanding the relationship between knowledge and African Americans' donation decision-making. Patient Educ Couns. 2008;70:242-250.

14. Haustein SV, Sellers MT. Factors associated with (un)willingness to be an organ donor: Importance of public exposure and knowledge. Clin Transplant. 2004;18:193-200. 
15. Rothschild ML. Marketing communications in nonbusiness situations or why it's so hard to sell brotherhood like soap. J Market. 1979;43:11-20.

16. Yuen CC, Burton W, Chiraseveenuprapund P, et al. Attitudes and beliefs about organ donation among different racial groups. $J$ Nat Med Assoc. 1998;90:13-18.

17. Morgan M, Mayblin M, Jones R. Ethnicity and registration as a kidney donor: The significance of identity and belonging. Soc Sci Med. 2008;66:147-158.

18. Morgan SE, Miller JK. Beyond the organ donor card: The effect of knowledge, attitudes, and values on willingness to communicate about organ donation to family members. Health Commun. 2001;14:121-134.

19. Alden DL, Cheung AH. Organ donation and culture: A comparison of Asian American and European American beliefs, attitudes, and behaviors. J Appl Soc Psychol. 2000;30:293-314.

20. Chapa J. Hispanics and organ donation: Prospects, obstacles and recommendations. In: Background Papers for the Surgeon General's Workshop on Organ Donation. Washington, DC: U.S. Public Health Service; 1991. Available at: http://profiles.nlm. nih.gov/NN/B/C/Z/J/_nnbczj.pdf.
21. McNamara P, Guadagnoli E, Evanisko MJ, et al. Correlates of support for organ donation among three ethnic groups. Clin Transplant. 1999;13:45-50.

22. Conesa C, Ríos A, Ramírez P, et al. Psychosocial profile in favor of organ donation. Transplant Proc. 2003;35:1276-1281.

23. Frates J, Bohrer G, Tomas D. Promoting organ donation to Hispanics: The role of media and medicine. J Health Commun. 2006;7:683-698.

24. Feld SL. Social structural determinants of similarity among associates. Am Soc Rev. 1982;47:797-801.

25. Stukas AA, Dew MA, Switzer GE, et al. PTSD in heart transplant recipients and their primary family caregivers. Psychosomatics. 1999;40:212-221.

26. Skinner C, Strecher V, Hospers H. Physicians' recommendations for mammography: Do tailored messages make a difference? Am J Public Health. 1994;84:43-49.

27. Bull FC, Kreuter MW, Scharff DP. Effects of tailored, personalized and general health messages on physical activity. Patient Educ Couns. 1999;36:181-192. 